\title{
NONLINEAR VIBRATION SIGNAL TRACKING OF LARGE OFFSHORE BRIDGE STAYED CABLE BASED ON PARTICLE FILTER
}

\author{
Qingwei Ye, Ph. D. \\ Zhimin Feng, Prof. \\ Debin Yuan, Ms. C. \\ Ningbo University, Zhejiang, China
}

\begin{abstract}
The stayed cables are key stress components of large offshore bridge. The fault detection of stayed cable is very important for safe of large offshore bridge. A particle filter model and algorithm of nonlinear vibration signal are used in this paper. Firstly, the particle filter model of stayed cable of large offshore bridge is created. Nonlinear dynamic model of the stayed-cable and beam coupling system is dispersed in temporal dimension by using the finite difference method. The discrete nonlinear vibration equations of any cable element are worked out. Secondly, a state equation of particle filter is fitted by least square algorithm from the discrete nonlinear vibration equations. So the particle filter algorithm can use the accurate state equations. Finally, the particle filter algorithm is used to filter the vibration signal of bridge stayed cable. According to the particle filter, the de-noised vibration signal can be tracked and be predicted for a short time accurately. Many experiments are done at some actual bridges. The simulation experiments and the actual experiments on the bridge stayed cables are all indicating that the particle filter algorithm in this paper has good performance and works stably.
\end{abstract}

Keywords: nonlinear vibration of stayed cables; large offshore bridge; particle filter; signal tracking

\section{INTRODUCTION}

Particle filter is known as sequential Monte Carlo method (SMC) in statistics. It is a sophisticated model estimation technique based on large rand simulations [8]. Particle filters are usually used to estimate nonlinear models, but typically where the state space of the latent variables is continuous rather than discrete. It is an advance filter tool compare with Kalman filter. They are often an alternative to the Extended Kalman filter (EKF) or unscented Kalman filter (UKF) with the advantage that, with sufficient samples, they approach the Bayesian optimal estimate, so they can be made more accurate than either the EKF or UKF. Particle filters have important applications in signal processing, econometrics and in other fields $[3,8]$.

As the main bearing member, stayed cables are the key stress components of cable-stayed bridge. Cable vibration signal can reflect the change of the external excitation and cable force timely [6,7]. For its light quality, flexibility of structure and low damping, stayed cables produce large amplitude vibration easily under external excitation. It really poses an extraordinary threat to the safety and durability of the cables $[10,11,12]$. Feature extraction of the cable vibration signal not only can reflect the bridge's running state, but also is the basis of follow-up parameters measurement. It has great significance in application engineering. Taking the sag influence and geometrical non-linearity into consideration, cable vibration is the mutual coupling of deck vibration and cable natural vibration, showing nonlinear vibration characteristics $[1,5]$. In order to solve the problem that nonlinear vibration equation is hard to solve and the nonlinear signal is difficult to process, the commonest numeric integration algorithms, such as Runge-Kutta, have worse anti-noise ability and high distortion under strong noise environment. Due to lacking of effective signal filtering methods, there is a great limitation in the field of dynamic signal analysis.

Based on cable-beam coupling nonlinear vibration theory, the article uses finite difference method to build discrete nonlinear vibration model. Then particle filter theory is applied in the filtering of the forecast signal, building a new method to solve nonlinear model problem. The simulation results and cable vibration signal tests show that particle filter theory combined with discrete nonlinear vibration model represses the noise effectively and extracts cable vibration signal accurately. It provides a stable and reliable signal tracking and forecasting method. 


\section{PARTICLE FILTER PRINCIPLE}

Particle filter theory $[3,8]$ is also called sequential MonteCarlo theory, condensation algorithm, boot strap filter algorithm and sampling importance resample, etc. By the means of looking for a group of random samples, spreading in state space, the theory replaces integration by sample mean through approximating the posterior probability density function. Particle filter is the estimation process getting state minimum variance. These samples are called particles. It realizes recursion Bayes estimation via nonparametric MonteCarlo method. PF theory estimates target state through the constantly change of particle weight in the state-space model.

The state equation and observation equation for common discrete system can be expressed as follows:

$$
x_{k+1}=f_{k}\left(x_{k}, v_{k}\right) \quad y_{k+1}=h_{k}\left(x_{k}, n_{k}\right)
$$

Where $\mathrm{x}_{\mathrm{k}}$ is the system state vector at $\mathrm{k}$ moment. $\mathrm{y}_{\mathrm{k}}$ is the observation output. And the $v_{k}$ is the system noise. The $n_{k}$ is the observation noise. The $f(\cdot)$ and $\mathrm{h}(\cdot)$ make up the system state equation and observed equation. $\mathrm{X}_{\mathrm{k}}=\left[\mathrm{x}_{0}, \mathrm{x}_{1}, \ldots, \mathrm{x}_{\mathrm{k}}\right]$ and $\mathrm{Y}_{\mathrm{k}}=\left[\mathrm{y}_{0}, \mathrm{y}_{1}, \ldots, \mathrm{y}_{\mathrm{k}}\right]$ are defined to be the accumulation state and accumulation observation by $\mathrm{k}$ moment.

If the initial probability density function is known as $p\left(x_{0} \mid y_{0}\right)=p\left(x_{0}\right)$ the state prediction equation is as follows. [3]

$$
p\left(x_{k} \mid x_{k-1}\right)=\int p\left(x_{k} \mid x_{k-1}\right) p\left(x_{k-1} \mid y_{1: k-1}\right) d x_{k-1}
$$

The status update equation can be expressed as

$$
p\left(x_{k} \mid y_{1: k}\right)=p\left(y_{k} \mid x_{k}\right) p\left(x_{k} \mid y_{1: k-1}\right) / p\left(y_{k} \mid y_{1: k-1}\right)
$$

Among the formula $p\left(y_{k} \mid y_{1: k-1}\right)=\int p\left(y_{k} \mid x_{k}\right) p\left(x_{k} \mid y_{1: k-1}\right) d x_{k}$

The above formula is the basic idea of optimal Bayesian estimation. Particle filter is sequential Monte-Carlo simulation method based on Bayes principle. If importance density function $q\left(x_{0: k} \mid y_{1: k}\right)$ is expressed in even product form as follows.

$$
q\left(x_{0: k} \mid y_{1: k}\right)=q\left(x_{0}\right) \prod_{j=1}^{k} q\left(x_{j} \mid x_{0: j-1}, y_{1: j}\right)
$$

And suppose state matching Markov process, under given state, weighted recursive formula can be expressed as follows:

$$
w_{k}=\frac{p\left(y_{1: k} \mid x_{0: k}\right) p\left(x_{0: k}\right)}{q\left(x_{k} \mid x_{0: k-1}, y_{1: k}\right) q\left(x_{0: k-1} \mid y_{1: k}\right)}=w_{k-1} \frac{p\left(y_{k} \mid x_{k}\right) p\left(x_{k} \mid x_{k-1}\right)}{q\left(x_{k} \mid x_{0: k-1}, y_{1: k}\right)}
$$

The simplest way to get important probability density function is making it equal to prior probability density:

$$
q\left(x_{k} \mid x_{0: k-1}, y_{1: k}\right)=p\left(x \mid x_{k-1}\right)
$$

If assuming that a group of random sample are got at $\mathrm{k}-1$ moment: $x_{k-1}^{i} \sim p\left(x_{k-1} \mid Y_{k-1}\right) \quad(\mathrm{i}=1 \ldots \mathrm{N}, \mathrm{N}$ is particle count), it can be obtained that:

$$
p\left(x_{k-1} \mid y_{1: k-1}\right) \approx \sum_{i=1}^{N} w_{k-1}^{i} \delta\left(x_{k-1}-x_{k-1}^{i}\right)
$$

Updated probability density function is expressed as degeneracy problem in particle filter.

$$
p\left(x_{k} \mid y_{1: k}\right) \approx \sum_{i=1}^{N} w_{k}^{i} \delta\left(x_{k}-x_{k}^{i}\right)=\frac{p\left(y_{k} \mid x_{k}\right) \sum_{i=1}^{N} w_{k-1}^{i} p\left(x_{k} \mid x_{k-1}^{i}\right)}{p\left(y_{k} \mid y_{1: k-1}\right)}
$$

Where, $\mathrm{N}_{\mathrm{eff}}$ is used to evaluate degradation level of a sample.

$$
N_{e f f}=1 / \sum_{j=1}^{N}\left(w^{j}\right)^{2}
$$

Among the formula $\mathrm{w}^{\mathrm{j}}$ is defined as normalized weight.

\section{NONLINEAR VIBRATION MODEL PROCESSING ALGORITHM BASED ON PARTICLE FILTER}

Due to non-parametric feature, particle filter overcome the shortage that random variable must obey Gaussian distribution in non-linear filter. It can express a much wider range of distribution than traditional models, and has strong ability to adapt to non-linear feature of variable parameters. Here, we try to apply particle filter theory in the processing of discrete nonlinear stayed cable vibration model. Based on the discrete nonlinear vibration equation, the system state equation and observation equation needed by particle filter are built. Finally, a new method, processing non-linear vibration model, is formed.

Taking the initial sag, geometric non-linearity and beam excitation into consideration, response of cable is non-linear vibration response.

Based on the assumptions as follows,

1) ignoring static deflection of beam

2) gravitational sag curve is parabola

3)cable vibration is the plane problem without regard to the vibration along the cable axial direction

4) cable is flexible and not concerned with flexural rigidity and shearing rigidity. The article analyses cable vibration system and builds cable-beam coupling model of cable-stayed bridge $[2,9]$.

Tagata [9] points out that the basic mode plays a major role in tightening string-end vibration. According to oscillatory differential equation [2] under beam-end excitation, combining with model boundary conditions, introducing viscous damping ratio $\zeta_{c}$, Galerkin method is used to get cable non-linear vibration equation of beam vertical excitation. In Fig.1, where $d_{1}$ is defined as span sag, $l_{c}$ is the length of cable. $\mathrm{v}_{\mathrm{b}}$ and $\mathrm{v}_{\mathrm{c}}$ are the beam lateral displacement and cable lateral deflection. 


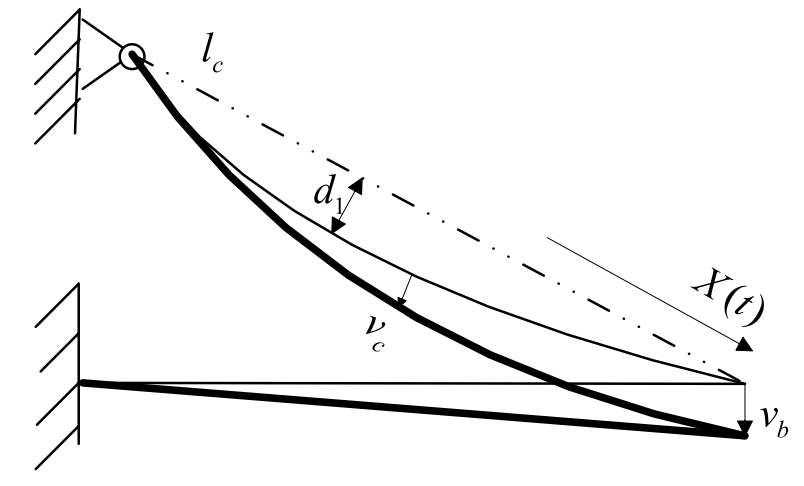

Fig. 1. Stayed cable model

$\ddot{x}(t)+b \dot{x}(t)+c_{1} x(t)+c_{2} \cos \left(w_{b} t\right) x(t)+d x^{2}(t)+e x^{3}(t)+f \cos \left(w_{b} t\right)=0(10)$

$a=\pi / l_{c}, b_{1}=d_{1} / l_{c}$ The coefficients in the formula can be expressed as:

$f=E_{c} A_{c} \frac{32 b_{1}}{m_{c} l_{c}^{2} \pi}, \quad b=2 \xi_{c} w_{c}, \quad c_{1}=w_{c}{ }^{2}, \quad d=\frac{E_{c} A_{c} a^{2} b_{1}}{m_{c} l_{c}}\left[\frac{64 a l_{c}}{3 \pi^{2}}+16 / \pi\right], \quad c_{2}=\phi \chi$

$\phi=\frac{E_{c} A_{c} a^{2}}{m_{c} l_{c}}\left[1-16 b_{1}^{2}\left(\frac{1}{6}-\frac{1}{\pi^{2}}\right)\right]-\frac{32 a b_{1}^{2}}{m_{c} l_{c}^{2} \pi}, \quad e=\frac{E_{c} A_{c}}{m_{c} l_{c}}\left[\frac{3}{8} a^{4} l_{c}-a^{4} b_{1}^{2} l_{c}\left(1-\frac{3}{2 \pi^{2}}\right)-\frac{10 a^{3} b_{1}^{2}}{\pi}\right]$

Where $\mathrm{x}(\mathrm{t})$ is the displacement function of stayed cable. $\mathrm{w}_{\mathrm{c}}$ is first natural frequency considering the sag influence and the initial equilibrium position. $\mathrm{w}_{\mathrm{b}}$ is cable oscillation frequency. $\mathrm{Z}$ is the vibration amplitude component along cable direction. $\mathrm{E}_{\mathrm{c}} \mathrm{A}_{\mathrm{c}}$ is the tensile rigidity of cable. $\mathrm{m}_{\mathrm{c}}$ is expressed as quality of unit length. $c_{2}$ and $\mathrm{f}$ indicate parameter vibration term and forced vibration term, reflecting non-linear feature of equation above.

In order to solve the non-linear equation above, traditional methods, such as Runge-Kutta, Adams multistep method, are often used. But interrelated solution models are too complex and the traditional methods have poor anti-noise capacity, and further, there is no subsequent filter algorithm matching with them. Based on finite difference theory, differentiation is replaced by central difference, as follows

$$
\dot{x}(k)=\frac{x(k+1)-x(k-1)}{2 h} \quad \ddot{x}(k)=\frac{x(k+1)-2 x(k)+x(k-1)}{h^{2}}
$$

Where represents the $k$-th sample of $x(t) . h$ is the quantifying step size. The less of $h$, the less of error, the difference value is more close to the differential value. The precision of $\dot{x}$ and $\ddot{x}$ can be ensured when sampling rate is big enough and $\mathrm{h}$ is small enough.

Eq.(11) is substituted in Eq.(10), discrete nonlinear vibration model on any cable position can be expressed as the next:

$$
\begin{gathered}
A x(k+2)+B_{1} x(k+1)+B_{2} \cos \left(w_{b} h(k+1)\right) x(k+1)+ \\
C x(k)+D x^{2}(k+1)+E x^{3}(k+1)+f \cos \left(w_{b} h(k+1)\right)=0
\end{gathered}
$$

Where $A=1 / h^{2}, B_{1}=c_{1}-2 / h^{2}, B_{2}=c_{2}, C=1 / h^{2}-b / 2 h$, $\mathrm{D}=\mathrm{d}, \mathrm{E}=\mathrm{e}$.

Knowing from the above formulas, if the cable model parameters $(\mathrm{A}, \mathrm{B} 1, \mathrm{~B} 2, \mathrm{C}, \mathrm{D}, \mathrm{E}, \mathrm{f})$ are known, based on the observation value of vibration signal at $\mathrm{k}$ moment and $\mathrm{k}+1$ moment, we can forecast vibration signal value at next moment effectively.

According to the discrete non-linear vibration model above, to estimate model parameters, Eq. (12) can be expressed in matrix operational form.

$$
Q_{k} \vec{r}=\vec{u}_{k+1}
$$

Where:

$$
Q_{k}=\left[\begin{array}{cccccc}
x_{k+1} & g_{k+1} * x_{k+1} & x_{k} & x_{k+1}^{2} & x_{k+1}^{3} & g_{k+1} \\
x_{k+2} & g_{k+2} * x_{k+2} & x_{k+1} & x_{k+2}^{2} & x_{k+2}^{3} & g_{k+2} \\
\vdots & \vdots & \vdots & \vdots & \vdots & \vdots \\
x_{k+N} & g_{k+N} * x_{k+N} & x_{k+N-1} & x_{k+N}^{2} & x_{k+N}^{3} & g_{k+N}
\end{array}\right]
$$

is defined as observation signal matrix. Constant matrix

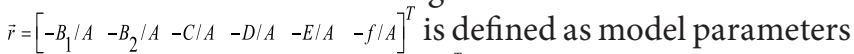
matrix. $\vec{u}_{k+1}=\left[\begin{array}{lllll}x_{k+2} & x_{k+3} & x_{k+4} & x_{k+5} \cdot x_{k+N+1}\end{array}\right]^{T}$ is defined as forecast signal matrix. $\mathrm{x}_{\mathrm{k}}=\mathrm{x}(\mathrm{k})$ is value of $\mathrm{x}(\mathrm{k})$ at k moment. $\mathrm{g}_{\mathrm{k}}=\cos \mathrm{w}_{\mathrm{b}} \mathrm{h}(\mathrm{k}-1)$ is a discrete cosine function. Random positive integer $\mathrm{N}$ represents expanding columns number.

Least-squares estimation(LSE) is used to get parameter matrix $\vec{r}$.For nonlinear vibration signals, forecast signal matrix $\vec{u}_{k}$ and observation signal matrix $\mathrm{Q}_{\mathrm{k}}$ can be built based on Eq. (13). Then the article uses LSE to get the estimate value $\hat{\vec{r}}$ of parameter matrix $\vec{r}$.

$$
\hat{\vec{r}}=\left(Q_{k}^{T} Q_{k}\right)^{-1} Q_{k}^{T} \vec{u}_{k}
$$

Knowing $\vec{r}$ from observation signal values, state equation of nonlinear stayed cable vibration model can be built from eq.(13). Adding observation noise $\vec{w}_{k}$ to state signal, observation equation can be got.The state equation and observation equation can be expressed as follows:

$$
\left\{\begin{array}{l}
\vec{u}_{k+1}=Q_{k} \stackrel{\hat{r}}{ }+\vec{v}_{k} \\
\vec{y}_{k+1}=\vec{u}_{k+1}+\vec{w}_{k}
\end{array}\right.
$$

Where, observation signal matrix $\mathrm{Q}_{\mathrm{k}}$ is seen as generalized state input at $\mathrm{k}$ moment. Forecast signal matrix $\vec{u}_{k+1}$ is regard as generalized state output at k+1 moment. $\vec{y}_{k+1}$ is defined as system observation output at $\mathrm{k}+1$ moment. $\vec{v}_{k}$ is state noise. $\vec{w}_{k}$ is the observation noise.

The state equation and observation equation upon can be seen as state equation and observation equation needed by particle filter. Following particle filter algorithm, based on signal observation values by this moment, we can predict the signal values in the follow-up moments. By calculating the weight of every particle and resample, the error between predicted value and truth value is being reduced during the filter process. The noise suppression purpose can be achieved effectively. 
According to the theory of discrete nonlinear model and particle filter upon, discrete nonlinear model processing method based on PF theory can be constructed as follows.

There is cable vibration signal from i moment to $\mathrm{j}$ moment. $X_{i \sim j}=\left[x_{i}, x_{i+1}, \cdots, x_{j}\right], j>i$ is defined as $\mathrm{i}$-th of vibration signal, with the length of $\mathrm{L}=\mathrm{j}-\mathrm{i}+1$. With PF theory and nonlinear vibration model, we can predict and track the signal of next moments.

(a) In the first place, for any observation signal $X_{k \sim k+N}$ with length of $\mathrm{L}=\mathrm{N}+1$, forecast signal matrix $\vec{u}_{k+1}$ and observation signal matrix $\mathrm{Q}_{\mathrm{k}}$ is built from eq.(13). Then following eq.(14), parameter matrix estimate value can be obtained by using Least-squares estimation method, as follows:

$$
\hat{\vec{r}}=\operatorname{LSE}\left(X_{k \rrbracket k+N}\right)=\left(Q_{k}^{T} Q_{k}\right)^{-1} Q_{k}^{T} \vec{u}_{k}
$$

(b) After knowing parameter matrix estimate value $\hat{\vec{r}}$ based on discrete nonlinear model and eq.(15), the state equation and observation equation needed by particle filter is built easily.

(c) Particle filter designed on the basis of Eq.(15) processes $\mathrm{k}$-th vibration signal $\mathrm{X}_{\mathrm{k} \sim(\mathrm{k}+\mathrm{N})}$, filtering out the environment noise, and get follow-up output signal values $\hat{y}_{k+N+1}$.

The resample method is defined as follows: calculating the weight of every particle: $q=\exp \left\{-\left(\hat{x}_{k}-\hat{y}_{k}\right)^{2} / 2 Q\right\}$.Where, $\hat{x}_{k}$ is represent observation value of $\mathrm{x}_{\mathrm{k}}$, which is obtained from nonlinear model immediately. $\hat{y}_{k}$ is defined as particle filter value of $x_{k}$, in which the noise is restrained effectively. Finally, we decide filter signal value based on normalized weight coefficients.

(d) After particle filter at $\mathrm{k}$ moment, predicted signal value $\hat{y}_{k+N+1}$ is equal to observation value $\mathrm{x}_{\mathrm{k}+\mathrm{N}+1}$ at $\mathrm{k}+\mathrm{N}+1$ moment. Using $\mathrm{x}_{\mathrm{k}+\mathrm{N}+1}$ to update vibration signal segment $X_{k+1 \sim(k+N+1)}$. The steps upon are repeated to update observation signal matrix $\mathrm{Q}_{\mathrm{k}+1}$ and forecast signal matrix $\vec{u}_{k+2}$. Loop forecast can get cable vibration signal of any length we need.

According to the process method of discrete nonlinear vibration model upon, we can draw the follows conclusions easily: In system state equation, generalized state inputs contains nonlinear terms. And there is obvious non-linear relation between state output, observation output and input. Particle filter can process the non-linear relation effectively. Combine with discrete non-linear cable model, particle filter can predict signal of any period time theoretically. It provides a convenience to anticipate stayed-cable performance in advance.

\section{SIMULATION EXPERIMENT}

In order to check the effect of particle filter theory applied on discrete non-linear vibration model, one stayed cable of a cable-stayed bridge was taken into research. According to article [12],the parameters are set as follows: $A_{c}=71.97 \times 10^{-4} \mathrm{~m}^{2}$, $\theta=56.4^{0}, 1_{c}=129.2 \mathrm{~m}$, the axial tensile force of cable $\mathrm{H}=33000 \mathrm{kN}$, quality of unit length $\mathrm{m}_{\mathrm{c}}=58.8 \mathrm{~kg} / \mathrm{m}$, modulus of elasticity $\mathrm{E}_{\mathrm{c}}=200 \mathrm{GPa}$ the effect of viscous damping ratios is taking into account, $\zeta_{\mathrm{c}}=0.002$. It can be proved easily that the nonlinear system including square term and cubic term can produce resonance only when $\mathrm{w}_{\mathrm{b}}=\mathrm{w}_{\mathrm{c}}$ or $\mathrm{w}_{\mathrm{b}}=2 \mathrm{w}_{\mathrm{c}}$. For the sake of simplifying the simulation process, the article do the experimental study only under the situation $\mathrm{w}_{\mathrm{b}}=2 \mathrm{w}_{\mathrm{c}}$.

According to finite difference theory, smaller the time discrete step $h$ is, higher precision of the discrete nonlinear model shown by Eq(12) is. Setting $h=0.001$ s, under different noise environment, the article extracts the vibration signal from discrete nonlinear system and compares the signal accuracy with classical Runge-Kutta method (under no noise environment).

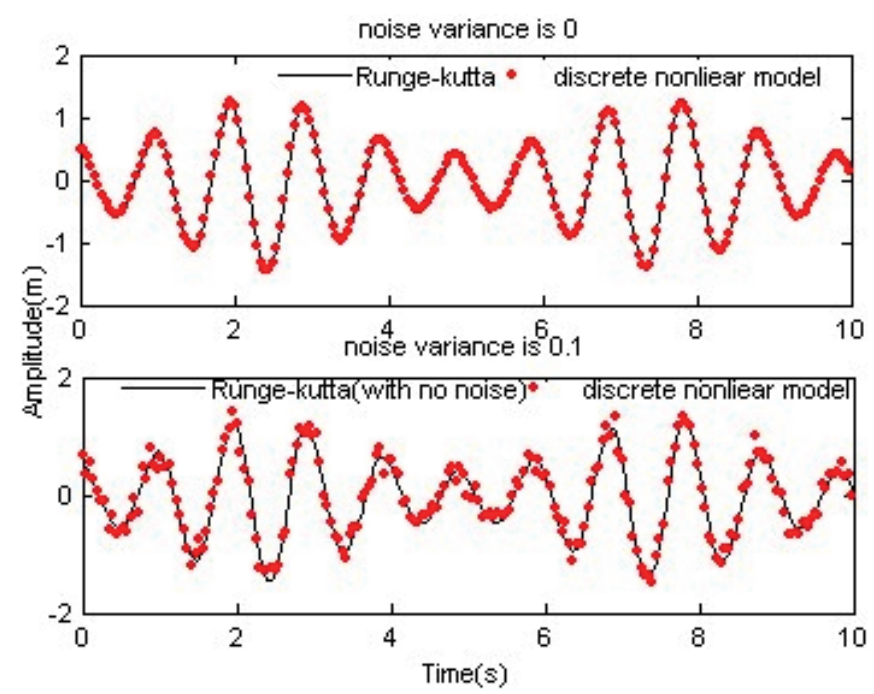

Fig. 2. The extracting efficiency based on discrete model

As we can see in Fig.2, with no noise or low noise, discrete nonlinear model built can be used to extract extremely high accurate signal. The degree of coupling between Vibration signal curve forecast by Runge-kutta theory and discrete nonlinear model is very high. Under the situation that the noise variance is equal to 0.1 , due to noise accumulation, the vibration signal, extracted by nonlinear model, compared with no noise signal (extracted by Runge-Kutta under no noise environment), happen serious distortion. SNR depress to $12.09 \mathrm{~dB}$.

Combined with Fig.1 example, the signal with noise (extracted by discrete nonlinear model under noise environment) from $1 \mathrm{~s}$ to $3 \mathrm{~s}$ is assume as observation signal. System noise $v_{k}$ variance is 0.001 . Observation noise variance $u_{k}$ is equal to 0.1 and 0.05 . Particles number is 500 . Observation signal length $\mathrm{N}$ is equal to 2000.

For convenient viewing, the signal between $3 \mathrm{~s}-5 \mathrm{~s}$ is forecasted by particle filter theory as Fig. 3 and Fig.4. Signal points with noise have large swing around pure signal. SNR is fall to $14.67 \mathrm{~dB}$ and $12.09 \mathrm{~dB}$. After being processed by particle filter, the signal obtained from nonlinear model shows satisfactory agreement with pure signal. SNR is up to $29.61 \mathrm{~dB}$ and $26.65 \mathrm{~dB}$. Predictive signal is highly accurate. The conclusion shows the application of particle filter can reduce effect of noise effectively and keep signal accuracy. Signal after being filtered is of great practical value in engineering. 


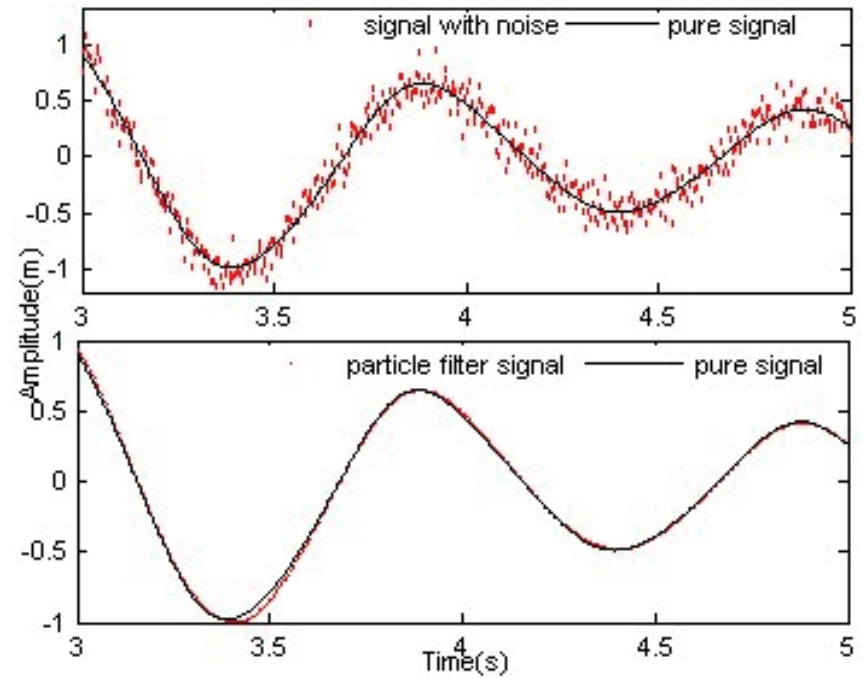

Fig. 3. The simulation analysis comparison at $\operatorname{VAR}(u k)=0.05$

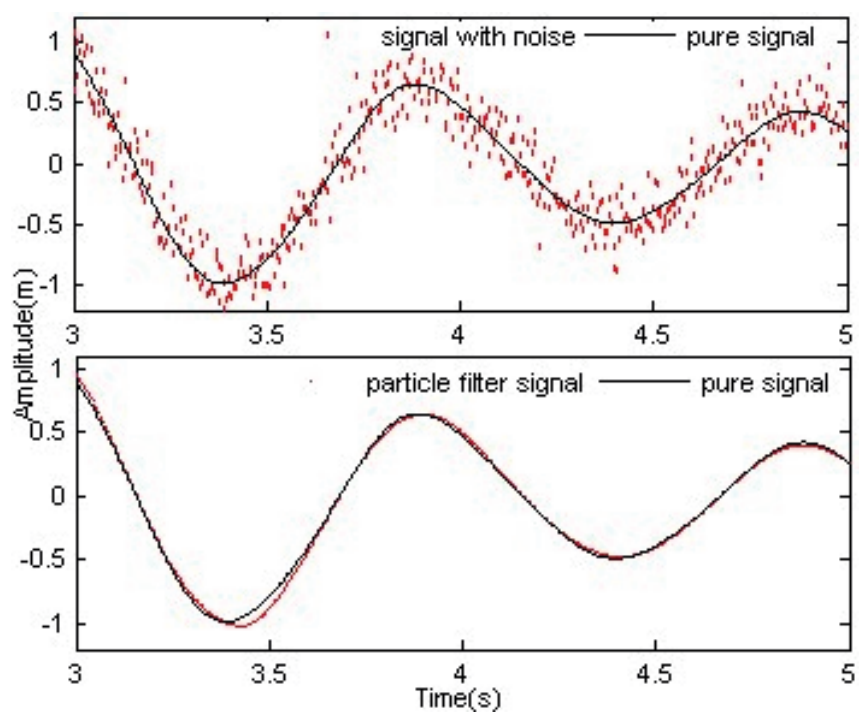

Fig. 4. The simulation analysis comparison $\operatorname{VAR}(u k)=0.1$

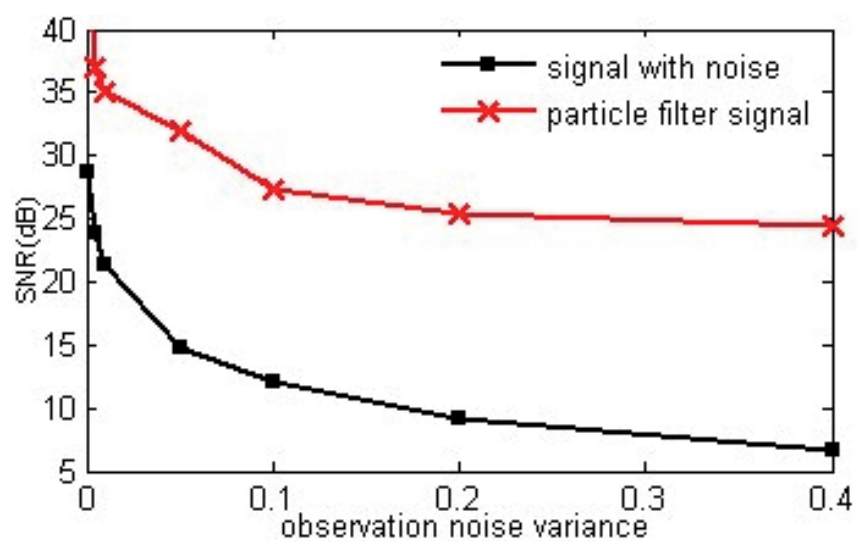

Fig. 5. The relation curve between noise variance and SNR
As presented in Fig5, with different observation noise variance, signal with noise (obtained from nonlinear model immediately) and practical filter signal (which with noise being filtered) have different performance. The relation curve of SNR and observation noise variance is shown as follows.

\section{THE APPLICATION IN CABLE-STAYED BRIDGE}

In the following test, it uses the predicted algorithm based on particle filter theory on the cable-stayed bridge. We use WS - ZHT2 vibration equipment and double sensors to collect the vibration signal. The sensors are installed as shown in Fig.6.

It tests acceleration response through the environmental stimulation (disturbed by strong winds and others). Signal collection scheme adopts double multi-input and single output pulse incentive; one sensor to collect vibration response signal, the other sensor to collect environmental stimulation signal. 4000 points for sampling points, sampling frequency is set as $100 \mathrm{~Hz}$. Based on particle filter and nonlinear model method, a signal segment for $5 \mathrm{~s}$ time length is seen as known signal point, to predict cable signal point ranged from $5 \mathrm{~s}-40 \mathrm{~s}$. Then it is compared with the signal date collected by the sensors. The effect is presented as Fig.7.

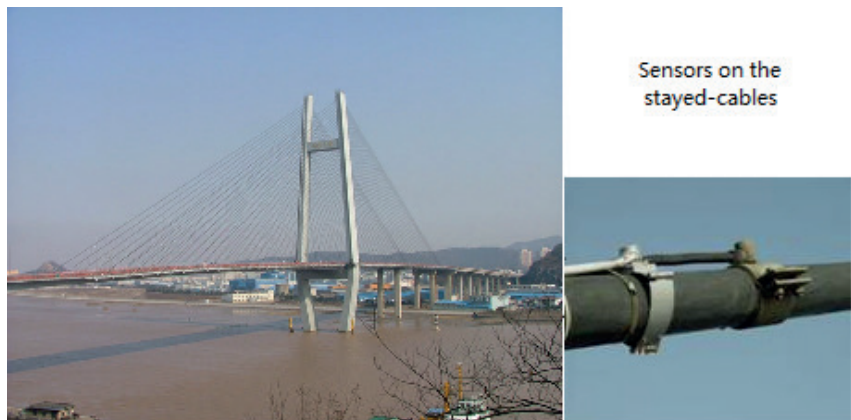

Fig. 6. The picture of bridge and experimental devices

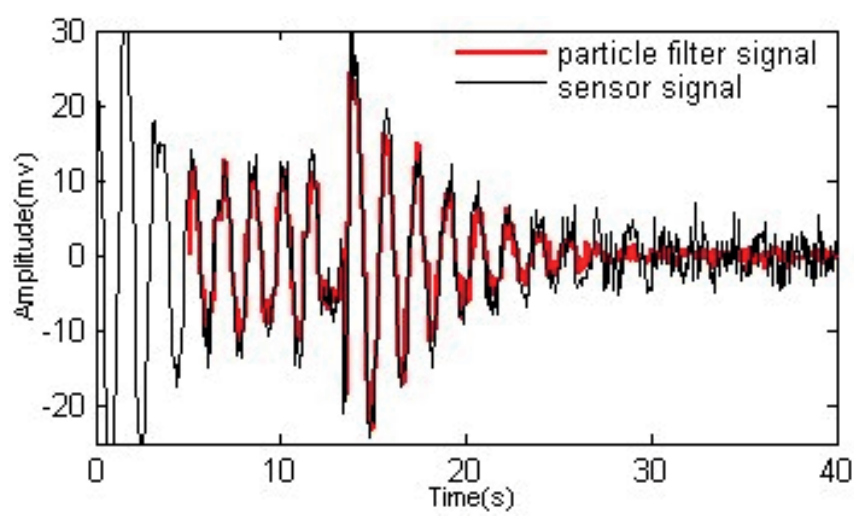

Fig.7. The particle filter effect of cable-stayed bridge data

The cable vibration signal filtered by particle filter, among the time from $25 \mathrm{~s}-40 \mathrm{~s}$, is compared with real cable signal. Vibration amplitude caused by environment noise is effectively restricted and mean decay to $32 \%$ of original. Among the 
whole particle filter period from $5 \mathrm{~s}$ to $40 \mathrm{~s}$, the amplitude of filtered signal present attenuation characteristics. As we can see from the signal features, particle filter theory combined with nonlinear model has well anti-noise ability and can improve signal precision effectively.

\section{CONCLUSIONS}

Based on finite difference theory, this article builds a discrete nonlinear cable vibration model and combines particle filter theory with it. The new cable signal processing method finally reaches the goal that noise impetus is weakened and accurate cable vibration signal is distilled. The particle filter algorithm has good applicability to cablestayed nonlinear model based on finite difference theory. The difficult to solve the cable nonlinear model, due to vibration model parameter nonlinearity, is solved effectively. The effective combination of particle filter with discrete model can overcome the high-distortion caused by noise accumulation and ensure accuracy of the prediction. Vibration signal after filter processing fully satisfy the need of engineering application, showing good potential in applications.

\section{ACKNOWLEDGEMENT}

This work has been financially supported by National Natural Sciences Funds of China (No. 61071198), Nature Science Funds of Zhejiang Province (No. LY13F010015) and Nature Science Funds of Ningbo (No. 2012A610019). This work is sponsored by K.C.Wong Magna Fund in Ningbo University.

\section{REFERENCES}

1. Chen Shuisheng, Sun Bingnan, "Numerical Study on Nonlinear Parametric Vibration of Coupled Cables and Bird Gedecks", Chinese Journal of Civil Engineering, Vol.36, No.4, pp.70-75, 2003.

2. Gattulli V, Morandini M, Paolone A, "Parametric Analytical Model for Non-Linear Dynamics in CableStayed Beam", Earthquake Engineering and Structural Dynamic, Vol.31, pp.1281-1300, 2002.

3. [3] Guillaume Thirel, Peter Salamon, Peter Burek and Milan Kalas. Assimilation of MODIS Snow Cover Area Data in a Distributed Hydrological Model Using the Particle Filter. Remote Sensing, Vol.5, No.11, pp.58255850, 2013.

4. Han Gao and Jingwen Li . "Detection and Tracking of a Moving Target Using SAR Images with the Particle FilterBased Track-Before-Detect Algorithm". Sensors, Vol.14, No.6, pp. 10829-10845, 2014.
5. Hong weiming, Gao lili, "Vibration Analysis of Nonlinear Coupling System of cable-stayed beam", Journal of Vibration Engineering, Vol.21, No.2, pp.115-119, 2008.

6. K.B.Waghulde, Dr. Bimlesh Kumar, "Vibration Analysis of Cantilever Smart Structure by using Piezoelectric Smart Material", International Journal on Smart Sensing and Intelligent Systems, Vol.4, No.3, pp.354-375, 2011.

7. Matsumoto M, Shiraishi N, Shirato H, "Rain-Wind Induced Vibration of Cables of Cable-Stayed Bridge". Journal of Wind Engineering and Industrial Aerodynamics, Vol.41, pp.2011-2022, 1992.

8. Olivier Brun, Vincent Teuliere, Jean-Marie Garcia, "Parallel Particle Filtering", Journal of Parallel and Distributed Computing, Vol.62, pp.1186-1202, 2002.

9. Tagata G. Harmonically Forced, "Finite Amplitude Vibration of a String", Journal of Sound and Vibration, Vol.51, No.4, pp.483-492, 1977.

10. Wang Zhigang, Sun Bingnan, "Parametric Vibration of Cable-Stayed Bridge", Chinese Journal of Civil Engineering, Vol.35, No.5, pp.28-33, 2002.

11. Ye Qingwei, Wang Tongqing, "Optimization modal analysis with PSO based on spectrum segmentation", Chinese Journal of Scientific Instrument, Vol.20, No.8, pp.1584-1590, 2009.

12. Zhao Yueyu, Lv Jiangen, "Non-linear Parametric Vibration of Cables in Cable-arch Composite Structures", Chinese Journal of Civil Engineering, Vol.39, No.12, pp.67-77, 2006.

\section{CONTACT WITH AUTHOR}

\author{
Qingwei Ye \\ Box 54, \\ Ningbo University \\ 315211 Zhejiang \\ China
}

tel.: $+85-13805877051$

rmail: personalfree@163.com 